\title{
Acquired Diaphragmatic Eventration in a Dog
}

\author{
Bruna Marquardt Lucio',2, Rafael Almeida Fighera ${ }^{2}$, Saulo Tadeu Lemos Pinto Filho ${ }^{3}$ \& Mariana Martins Flores ${ }^{2}$
}

\begin{abstract}
Background: Diaphragmatic eventration is characterized by weakness of the diaphragmatic muscle, which leads to cranial dislocation of the affected diaphragm and, ultimately, in dyspnea. This condition is rare in humans and even rarer in animals, and may be congenital or acquired. The acquired form is less commom and may be induced by trauma or inflammation and neoplastic invasion of the phrenic nerve. Here, we report a case of acquired diaphragmatic eventration in a dog, with the aim of increasing the knowledge of this condition in animals and helping others to recognize and treat future cases.

Case: A 12-year-old male dachshund presented with severe dyspnea, exercise intolerance and episodes of coughing. Based on a physical examination and imaging, the main suspicion was a diaphragmatic hernia, and surgery was performed. When the surgeon entered the thoracic cavity, an extremely thin - yet, intact - right hemidiaphragm was observed. The left side of the diaphragm was normal. A polypropylene mesh was sutured to the affected diaphragm in an attempt to strengthen the hemidiaphragmatic muscles and prevent further insinuations of viscera into the thoracic cavity. The dog developed bronchopneumonia, postoperatively, and was hospitalized and treated with antibiotics, analgesics and support medication. However, the dog died five days after surgery. A postmortem examination revealed that the right side of the diaphragm was markedly thin and flaccid. Diaphragm samples were collected for histopathological examination. For comparison, a sample of normal diaphragm was collected from a same age, matched dachshund that died due to an unrelated condition. This tissue was called "diaphragm control", and it was collected in order to compare the histologic features of a normal diaphragm muscle with the affected one. Histopathology revealed a marked reduction of muscle fibers. In the affected sample, replacement of these fibers by fibrous connective tissue and a marked infiltration of fat were seen among the remaining muscle fibers. Multifocal areas of necrosis were also observed affecting some muscles fibers. Microscopic comparisons of both diaphragm samples (affected $v s$. control) revealed a drastic difference in the amount of muscle fibers and fat, corroborating the intense diaphragmatic atrophy observed in the diaphragm from the affected dog. Based on clinical presentation, the gross lesions observed during surgery and later during the post mortem examination, and histopathological findings, a definitive diagnosis of acquired diaphragmatic eventration was established.

Discussion: Diaphragmatic eventration is rarely reported in small animal clinics and thus may be confused with other conditions. It must be mainly differentiated from diaphragmatic hernia and should be considered as a differential diagnosis when an animal, regardless of age, presents with dyspnea, apathy and coughing episodes. On suspecting diaphragmatic eventration, surgical intervention should be carried out as soon as possible. The recommended treatment is plication of the affected hemidiaphragm. However, in the present case, a polypropylene mesh was sutured to the affected area to support the atrophic muscles. It is thought that, trauma injured the dog's phrenic nerve, affecting right hemidiaphragmatic innervation, and generating progressive atrophy of diaphragmatic muscle fibers. Subsequently, the dog developed diaphragmatic eventration due to diaphragmatic fragility. Diaphragmatic eventration is a very rare disorder in small animals and can be difficult to diagnose based solely on physical and radiographic examinations.
\end{abstract}

Keywords: diaphragm, dyspnea, canine, muscle atrophy, eventration, histopathology.

Descritores: diafragma, dipsneia, canino, atrofia muscular, eventração, histopatologia. 


\section{INTRODUCTION}

The condition diaphragmatic eventration is characterized by weakness of the diaphragmatic muscle, which leads to a cranial dislocation of the affected diaphragm [5]. Such a dislocation of the diaphragm leads to the formation of sacculation, which invades the thoracic cavity and may contain abdominal viscera [7]. The extent of muscle weakness in diaphragmatic eventration ranges from small, and inconspicuous to half of the diaphragm (hemidiaphragm) [6].

Diaphragmatic eventration is rare in humans, affecting approximately $0.001 \%-0.003 \%$ of births. It is even rarer in animals, having been described only in golden lion tamarins (Leontopithecus rosalia), a leopard (Panthera pardus), a horse foal, two young cats [3] and a dog [7].

Diaphragmatic eventration may be congenital or acquired. The former is characterized by muscle paralysis, atrophy, or aplasia caused by incomplete growth of muscle fibers during the embryonic period [4]. According to Kearns et al. [5], this is the most common form. The acquired form may be induced by trauma or inflammation and neoplastic invasion of the phrenic nerve [3].

Here, we report a case of acquired diaphragmatic eventration in a dog. To the best of our knowledge, this is the second report of this condition in canines. The aim of this report is to improve knowledge on acquired diaphragmatic eventration in animals and to others to recognize and treat future cases.

\section{CASE}

A 12-year-old male dachshund presented with severe dyspnea, exercise intolerance and episodes of coughing (Figure1). The animal shown clinical signs for 2 weeks, but the symptoms had worsened in the previous 2 days. The dog had no history of recent trauma, but had been run over by a car approximately eight years earlier. Physical examination revealed severe dyspnea, pale pink oral and ocular membranes and mild dehydration.

Blood count and biochemical function tests were normal. Thoracic radiography images were suggestive of diaphragmatic hernia, characterized by a portion of the liver that seemed to invade the thoracic cavity. From the images, it was not possible to determine if the diaphragm line was intact. The presence of the liver that seemed to invade the thoracic cavity was confirmed by ultrasonography.
Surgery for a diaphragmatic hernia repair was initiated immediately. However, when the surgeon entered the thoracic cavity, an extremely thin - yet, intact - right hemidiaphragm was observed. The right portion of the liver was adhered to the affected hemidiaphragm. This thin structure was characterized by a highly fragile membrane that ruptured when manipulated. After liver adherence was undone, a polypropylene mesh was sutured to the affected diaphragm in an attempt to strengthen the hemidiaphragmatic muscles and prevent further insinuation of viscera into the thoracic cavity. The left side of the diaphragm was normal.

After surgery, the dog was hospitalized for five days and received antibiotics, analgesics, and support medication. During this period, the dog also received intravenous fluid (Lactated Ringer's solution) ${ }^{1}$ and was fed a high calorie diet $\left(\text { Recovery }{ }^{\circledR}\right)^{2}$. When severe dyspnea occurred, the dog was put on oxygen therapy. The medication and support care was performed continuously during the 2 days prior to death. The animal died five days after surgery.

On a post mortem examination, the right side of the diaphragm was found to be markedly thin and flaccid (Figure 2). The $7 \times 7 \mathrm{~cm}$ polypropylene mesh was still attached to the right diaphragm. The lungs were diffusely firm and dark red.

Diaphragm and lung samples were collected for histopathological examination and fixed in $10 \%$ buffered formalin. Samples were processed, embedded in paraffin, cut, stained with haematoxylin and eosin and examined under a light microscope. For comparison, a sample of normal diaphragm was collected from an age-matched dachshund that who died due to an unrelated condition. The control tissue samples were prepared in an identical manner.

A microscopic examination revealed a marked atrophy of muscle fibers in the affected diaphragm. Replacement of these fibers by fibrous connective tissue and a marked infiltration of fat were seen among the remaining muscle fibers. Multifocal areas of necrosis were also observed affecting some muscles fibers. Microscopic comparisons of both diaphragms (affected $v s$. control) revealed a drastic difference in the amount of muscle fibers and fat between the two samples, corroborating the intense diaphragmatic atrophy observed in the diaphragm from the affected dog (Figure 3). By contrast, in the left antimere, of the affected dog, only mild atrophy of the muscle fibers 
was seen. In this portion, atrophy was characterized more by a decrease in thickness of the muscle fibers than by a decrease in the number of fibers.

A histological evaluation of the lungs revealed large amounts of neutrophils, macrophages and cellular debris within alveolar, bronchial and bronchiolar lumens. Eosinophilic material - interpreted as aspirated material - was observed multifocally within the alveolar lumen. These findings support the diagnosis of suppurative bronchopneumonia due to aspiration. Because of impaired respiratory function, the dog probably aspirated exogenous material while eating or drinking. The resulting this severe inflammation of the lungs caused respiratory failure, ultimately leading to the animal's death.

Based on clinical presentation, the gross lesions observed during surgery and later in the post mortem examination, and histopathological findings, a definitive diagnosis of acquired diaphragmatic eventration was established.

\section{DISCUSSION}

Diaphragmatic eventration is rare in dogs and may be confused with conditions such as phrenic nerve paralysis, diaphragmatic hernia or congenital muscular deficiency of the diaphragm [7]. As this condition occurs rarely in small animal clinics, diaphragmatic hernia was initially suspected; however, this diagnosis was rejected at the time of surgery.

The clinical signs observed in this dog are similar to those described in the literature. Dyspnea in animals may be caused by a decrease in pulmonary functional capacity associated with abnormal diaphragm movement [5]. Gombac et al. [3] reported a case of two young cats with congenital diaphragmatic eventration that presented with severe dyspnea, cyanosis and apathy. In humans, respiratory distress has been reported in infants with diaphragmatic herniation, in older children and adults, this condition may be asymptomatic [1].

The recommended treatment for diaphragmatic eventration is plication of the affected hemidiaphragm [7]. However, in the present case, a polypropylene mesh was sutured to the affected area to provide greater support to the atrophic muscles. Such material has been used successfully to correct of anatomical defects in animals, as its crystalline molecular structure provides excellent tensile strength [2].
The lesions observed on necropsy and histopathological examination are consistent with those reported by Gombac et al. in cats [3], namely, fragile and thin diaphragmatic muscles, with the affected portions protruding into the thoracic cavity and marked muscular atrophy.

In the present case, it is believed that, past trauma (described by the dog's owner - but not evident on examination) had injured the dog's phrenic nerve, affecting right hemidiaphragmatic innervation, and generating progressive atrophy of diaphragmatic muscle fibers. Ultimately, the dog developed diaphragmatic eventration due to diaphragmatic fragility. In this case, the clinical signs appeared several years after the trauma.

Diaphragmatic eventration is a very rare disorder in small animals and can be difficult to diagnose based solely on physical and radiographic examina-

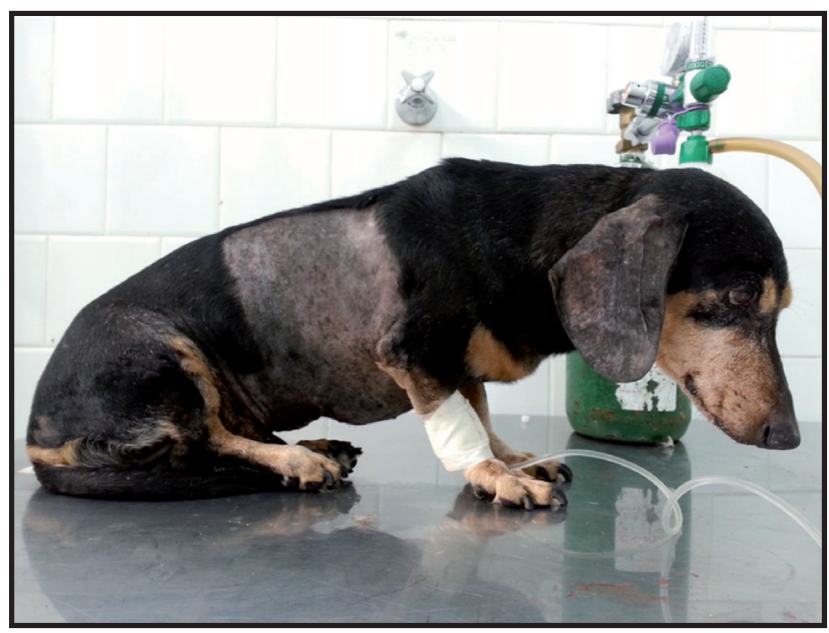

Figure 1. A 12-year-old male dachshund with severe dyspnea, exercise intolerance and episodes of coughing.

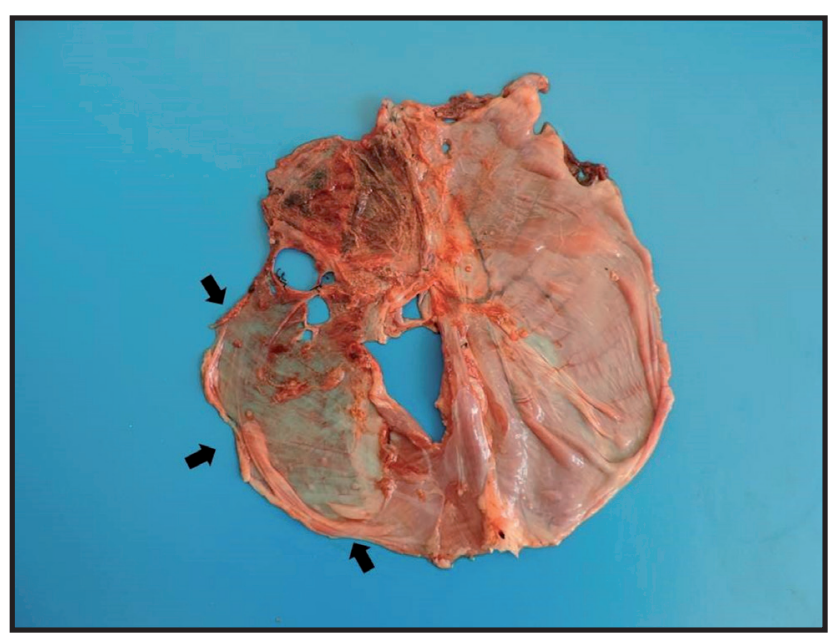

Figure 2. Right hemidiaphragm of the affected dachshund. Note the thin, flaccid and translucent portion of the diaphragm (arrows) compared to the normal side. 


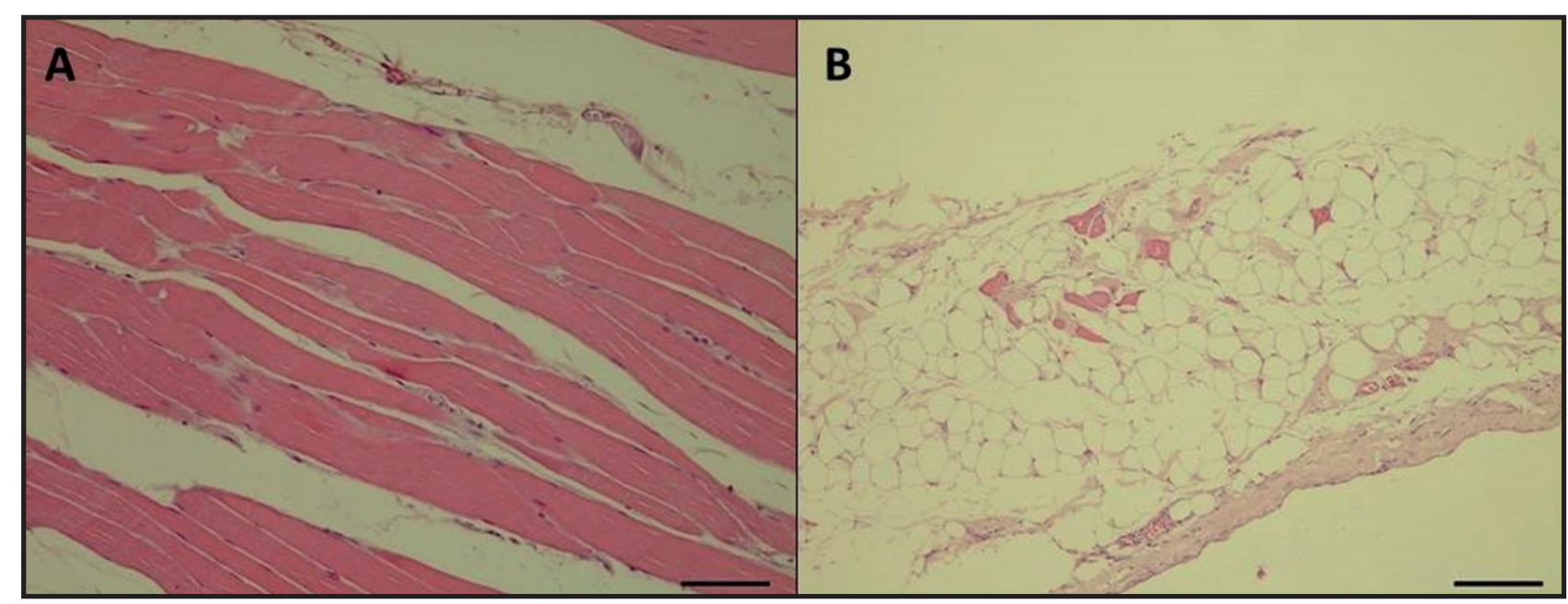

Figure 3. A- Normal diaphragm of an age-matched dachshund. The image shows a predominance of muscle fibers intermingled with little fat. B- Right hemidiaphragm of the dachshund with acquired diaphragmatic eventration. Note the intense adipose tissue infiltration with almost no visible muscle fibers, indicating severe muscular atrophy.

tions. Diaphragmatic eventration must be differentiated from diaphragmatic hernia and should be considered as a differential diagnosis when an animal, regardless of age, presents with dyspnea, apathy and coughing episodes. On suspecting diaphragmatic eventration, surgery should be performed as soon as possible.

\section{MANUFACTURERS}

${ }^{1}$ Baxter Hospitalar Ltda. São Paulo, SP, Brazil. ${ }^{2}$ Royal Canin Brazil. São Paulo, SP, Brazil.

Declaration of interest. The authors report no conflicts of interest. The authors alone are responsible for the content and writing of the paper.

\section{REFERENCES}

1 Anshu S., Poonam S., Singh R.J. \& Veena S. 2005. Eventration of Diaphragm - Embryological basis. Journal of the Anatomical Society of India. 54(2): 1-9.

2 Bojrab M.J. 2005. Técnicas Atuais em Cirurgia de Pequenos Animais. 3.ed. São Paulo: Roca, 920p.

3 Gombac M., Vreci M. \& Svara T. 2011. Congenital diaphragmatic eventration in two closely related British Shorthair cats. Journal of Feline Medicine and Surgery. 13(4): 276-279.

4 Jackson C., Collyer P.B. \& Loynachan A. 2006. Congenital diaphragmatic eventration in a stillborn foal. Journal of Veterinary Diagnostic Investigation. 18(4): 412-415

5 Kearns K.S., Jones M.P., Bright R.M., Toal R., Denovo R. \& Orosz S. 2000. Hiatal hernia and diaphragmatic eventration in a leopard (Panthera pardus). Journal of Zoo and Wildlife Medicine. 31(3): 379-382.

6 Pober B.R. 2007. Overview of epidemiology, genetics, birth defects and chromosome abnormalities associated with CDH. American Journal of Medical Genetics. 145C(2): 158-171.

7 Souza M.V., Malm C., Gheller V.A., Oliveira H.P., Menezes J.M.C. \& Bertolini C.M. 2004. Eventração diafragmática em cão. In: Congresso do Colégio Brasileiro de Cirurgia e Anestesiologia Veterinária (Indaiatuba, Brasil). p.118. 